\title{
On Boundary Values of Hypergeometric Functions of Several Variables
}

\author{
by
}

Kyoji SAITO*

In this note we shall give a brief sketch of the theory of the uniformization of complements of discriminants $D$ in $C^{n}$.

Let $D \subset C^{n}$ be a suitable hypersurface of $C^{n}$ which we shall refer to as a discriminant. Our main problem is (1) firstly to construct a kind of covering space $H$ of $C^{n}-D$ by making use of the solutions of some system of partial differential equations called the system of uniformization equations, and secondly to construct the inverse map from $H$ to a quotient space of $C^{n}-D$ by making use of some kind of Eisenstein series. The classical theory of elliptic integrals and elliptic modular functions can be reconstructed in this way. Thus our theory can be considered as a generalization of it for the theory of functions of several variables. In our approach to the problem, the theory of forms with logarithmic poles would play the fundamental role.

The results of the first half of this note is already published in [2]. So we refer to the note, for such as general theory of logarithmic forms. The detailed version of this note will be published elsewhere.

\section{$\S 1$ Logarithmic Forms}

Let $h\left(x_{1}, \cdots, x_{n}\right)$ be a reduced weighted homogeneous polynomial such that $h\left(t^{m_{1}} x_{1}, \cdots, t^{m_{n}} x_{n}\right)=t^{m} h\left(x_{1}, \cdots, x_{n}\right)$ with $m, m_{1}, \cdots, m_{n} \in \mathbf{N}$, and let $D$ be the hypersurface in $\boldsymbol{C}^{n}$ defined by the equation $h=0$.

Then we define:

Received October 12, 1976.

* Department of Mathematics, College of General Education, University of Tokyo, Komaba, Meguro, Tokyo, Japan. 


\section{Definition.}

$\Omega_{\boldsymbol{C}^{n}}^{1}(\log D)=\left\{\omega\right.$; germ of meromorphic 1 -form on $\mathbb{C}^{n}, \omega$ and $d \omega$ have only simple poles along $D\}$

$\operatorname{Der}_{\boldsymbol{C}^{n}}(\log D)=\left\{\delta:\right.$ germ of holomorphic vector field on $\boldsymbol{C}^{n} ; \delta h$ is divisible by $h\}$.

We know that each one is a dual $\mathcal{O}_{\boldsymbol{C}^{n}}$-module of the other.

Remark. In general these modules are not free. It is conjectured

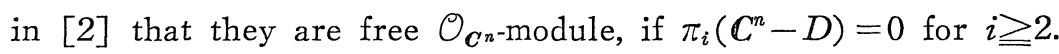

Example. The most important examples of such divisers are constructed by the use of reflection groups. For the notions of reflection groups and invariant polynomials we refer to Bourbaki [1].

Let $V$ be an $n$-dimensional real vector space and $G$ be a finite subgroup of $G L(V)$ generated by reflections. Let $R$ be the invariant subalgebra of the symmetric algebra $S(V)$ of $V$, and $P_{1}, \cdots, P_{n} \in R$ be a generator system of $R$ such as $R=\boldsymbol{R}\left[P_{1}, \cdots, P_{n}\right]$.

Let $\Delta \in S(V)$ be the product of linear functions defining the hyperplanes of reflections of $G$. Then $\Delta^{2} \in R$ can be represented as a polynomial of $P_{1}, \cdots, P_{n}$.

Now let us consider the complex Euclidean space $E=\left(V^{*} / G\right)^{\boldsymbol{C}}$ the complexification of the quotient space of $V^{*}$ by $G$, whose coordinate ring is $R \otimes C . \quad \Delta^{2}$ defines a hypersurface $D_{G}$ in $E$, which we call the discriminant of the reflection group $G$. Then a free basis $\omega_{i}$, and $X^{i}, i=1, \cdots, n$ for $\Omega_{E}{ }^{1}\left(\log D_{G}\right)$ and $\operatorname{Der}_{E}\left(\log D_{G}\right)$ can be constructed as follows.

Let $B(\cdot, \cdot)$ and $B^{\prime}(\cdot, \cdot)$ be the $G$ invariant quadratic forms on $V$ and $V^{*}$, which are positive definite. We may regard them as quadratic forms on the cotangential bundle $T_{V^{*}}^{*}$ and tangential bundle $T_{V^{*}}$ over $V^{*}$. Then $X^{i}=B\left(d p_{i}, \cdot\right)$, and $\omega_{i}=B^{\prime}\left(\frac{\partial}{\partial p_{i}}, \cdot\right)$ are vector fields and meromorphic 1 -forms on $V^{*}$ such as $X^{i} \cdot \omega_{j}=\delta_{j}{ }^{i}$. Since they are $G$-invariant, they induce vector fields and meromorphic 1 -forms on $E$, which are the required basis.

It is known that the complements of the discriminant loci of reflection groups are Eilenberg-MacLane spaces due to V. I. Arnold, E. Brieskorn, 
and P. Deligne.

Let $\omega_{1}=(1 / m)(d h / h), \cdots, \omega_{n}$ be a free basis for $\Omega_{\boldsymbol{C}^{n}}^{1}(\log D)$ and $X^{1}=\sum^{n} m_{i} x_{i} \frac{\partial}{\partial x_{i}}, \cdots, X^{n}$ be the dual basis for $\operatorname{Der}_{\boldsymbol{C}^{n}}(\log D)$. Then the exterior differentiation is given by $d=\sum_{i=1}^{n} \omega_{i} X^{1}$ and $\omega_{1} \wedge \cdots \wedge \omega_{n}=\frac{d x_{1} \wedge \cdots \wedge d x_{n}}{h}$.

Since $\Omega_{\boldsymbol{C}^{n}}^{1}(\log D)$ is a free $\mathcal{O}_{\boldsymbol{C}^{n} \text {-module, it corresponds to a vector }}$ bundle of rank $n$ over $\boldsymbol{C}^{n}$, which we shall denote by $T_{\boldsymbol{C}^{n}}^{*}(\log D)$. The inclusion of the sheaf $\Omega_{\boldsymbol{C}^{n}}^{1}$ of holomorphic one forms on $\mathbb{C}^{n}$ into $\Omega_{\boldsymbol{C}^{n}}^{1}(\log D)$ corresponds to a bundle homomorphism $i: T_{\boldsymbol{C}^{n}}^{*} \rightarrow T_{\boldsymbol{C}^{n}}^{*}(\log D)$, where $T_{\boldsymbol{C}^{n}}^{*}$ is the cotangential bundle of $\mathbb{C}^{n}$. Notice that $i$ induces a bundle isomorphism over $C^{n}-D$. Let us consider $\operatorname{Ker}(i)$ as a subvariety of $T_{\boldsymbol{C}^{n}}^{*}$, which we denote by $L(\log D)$ and call it the Lagrangean subvariety of logarithmic poles along $D . L(\log D)$ is nothing another than the subvariety of $T_{\boldsymbol{C}^{n}}^{*}$ defined by the common zeros of the principal symbols $\sigma\left(X^{i}\right), i=1, \cdots, n$.

We can show that $L(\log D)$ is a pure $n$-dimensional subvariety of $T_{\boldsymbol{C}^{n}}^{*}$, such that the restriction to $L$ of the symplectic form $\sum_{i=1}^{n} d x_{i} \wedge d \xi_{i}$ on $T_{\boldsymbol{C}^{n}}^{*}$, vanishes identically.

Since $h$ is a weighted homogeneous polynomial, $\Omega_{\boldsymbol{C}^{n}}^{1}(\log D)$ is a graded module in a natural way. We may assume that $\omega_{1}, \cdots, \omega_{n}$ and $X^{1}, \cdots, X^{n}$ are homogeneous. Put $-\operatorname{deg} \omega_{i}=\operatorname{deg} X^{i}=d_{i}$. Then we have $\sum_{i=1}^{n} d_{i}+\sum_{i=1}^{n} m_{i}$ $=m$.

Let us suppose $0=d_{1}<d_{2} \leqq \cdots \leqq d_{n}$, which is equivalent to the fact that $h$ cannot be represented as a product of the form $h_{1}\left(x_{1}, \cdots, x_{k}\right)$ $\times h_{2}\left(x_{k+1}, \cdots, x_{n}\right)$ by some change of coordinates.

\section{§ 2. The System of Uniformization Equations with respect to $\nabla$}

Now let us introduce a concept of connections with logarithmic poles.

\section{Definition.}

I. A connection $\nabla$ with logarithmic poles along $D$ is a sheaf homomorphism

$$
\nabla: \Omega_{\boldsymbol{C}^{n}}^{1}(\log D) \rightarrow \Omega_{\boldsymbol{C}^{n}}^{1}(\log D) \otimes \Omega_{\boldsymbol{C}^{n}}^{1}(\log D)
$$

with 
i) $\nabla\left(\omega+\omega^{\prime}\right)=\nabla \omega+\nabla \omega^{\prime}$

ii) $\nabla(f \omega)=d f \otimes \omega+f \nabla \omega$

II. $\nabla$ is integrable, if the following composition is zero.

$$
\Omega_{\boldsymbol{C}^{n}}^{1}(\log D) \rightarrow \Omega_{\boldsymbol{C}^{n}}^{1}(\log D) \otimes \Omega_{\boldsymbol{C}^{n}}^{1}(\log D) \rightarrow \Omega_{\boldsymbol{C}^{n}}^{2}(\log D) \otimes \Omega_{\boldsymbol{C}^{n}}^{1}(\log D) .
$$

III. $\nabla$ is torsion free, if the composition

$$
\Omega_{\boldsymbol{C}^{n}}^{1}(\log D) \rightarrow \Omega_{\boldsymbol{C}^{n}}^{1}(\log D) \otimes \Omega_{\boldsymbol{C}^{n}}^{1}(\log D) \rightarrow \bigwedge^{2} \Omega_{\boldsymbol{C}^{n}}^{1}(\log D)=\Omega_{\boldsymbol{C}^{n}}^{2}(\log D) .
$$

coincides with the exterior differentiation $d$.

IV. $\nabla$ is homogeneous, if it is a homogeneous morphism with respect to the canonical graduation.

We denote by $U\left(C^{n}, D\right)$ the set of all integrable torsion free homogeneous connections with logarithmic poles along $D$. It is easy to see that $U\left(C^{n}, D\right)$ has the structure of a finite dimensional algebraic variety.

For $\nabla \in U\left(C^{n}, D\right)$, let us determine the coefficients $\omega_{i}^{j}$ and $\Gamma_{i}^{j k}$ with respect to the basis $\omega_{i}$.

$$
\nabla \omega_{i}=\sum_{j=1}^{n} \omega_{i}^{j} \otimes \omega_{j}, \quad \omega_{i}^{j}=\sum_{k=1}^{n} \Gamma_{i}^{j k} \omega_{k} .
$$

Then $\Gamma_{i}^{j k}$ is a homogeneous function of degree $d_{j}+d_{k}-d_{i}$ on $\boldsymbol{C}^{n}$ satisfying some conditions coming from integrability and torsionfreeness of $\nabla$. Since $\sum_{i=1}^{n} \omega_{i}{ }^{i}$ is a closed form of degree zero, it has a presentation $\sum_{i=1}^{n} \omega_{i}{ }^{i}$ $=\sum c_{j} \frac{d h_{j}}{h_{j}}$ where $c_{j}$ are cnstants and $h_{j}$ are irreducible components of $h$.

From the torsion free condition, we obtain the following.

A logarithmic form $\omega$ is horizontal (i.e., $\nabla \omega=0$ ) iff there exists a function $u$ such that

i) $\quad \omega=d u=\sum_{i=1}^{n} X^{i} u \cdot \omega_{i}$, and

ii) $u$ satisfies the equations:

$$
X^{k} X^{j} u+\sum_{i=1}^{n} \Gamma_{i}^{j k} X^{i} u=0 \quad \text { for } j, k=1, \cdots, n .
$$

We call the above system of differential equations, the system of uniformization equations with respect to $\nabla$.

Since the principal symbol ideal of the equation is generated by $\sigma\left(X^{k}\right)$ $\times \sigma\left(X^{j}\right), k, j=1, \cdots, n$, the characteristic variety of the system coincides with the Lagrangean variety $L(\log D)$ of logarithmic poles along $D$. This 
shows the system of uniformization equations is a holonomic system.

From the integrability condition, the space of all solutions of the system of uniformization equations, denoted by $U_{\nabla}$, form $n+1$-dimensional complex vector space, including constant solutions.

Any element $u$ of $U_{\nabla}$ can be considered as a holomorphic function element on $C^{n}-D$, which is analyticaly continuable along any curve $C$ in $C^{n}-D$ so far as $C$ does not meet with the discriminant. Thus $u$ determins a univalent function on the universal covering space $\overparen{C^{n}-D}$ of $C^{n}-D$, which we denote again by the same notation $u$.

For a point $\tilde{x}$ of $\widetilde{C^{n}-D}$, the correspondence $U_{\nabla} \ni u \mid \rightarrow u(\widetilde{x}) \in C$ is a $C$-linear map, which gives an element of the dual space $U_{\nabla}{ }^{*}$, denoted by $u(\tilde{x}) \in U^{*}$. Since the constant function 1 of $U_{\nabla}$ takes the constant value 1 for any $\widetilde{x}$, the image of the map $u$ is contained in a hyperplane $V_{\nabla}{ }^{*}$ of $U_{\nabla}^{*}$.

The Jacobian of the map $u: \overparen{C^{n}-D} \rightarrow V^{*}$ can be calculated as follows.

Let $u_{1}, \cdots, u_{n}, 1$ be a basis for $U_{\nabla}$. Then

$$
\text { Jac. } \begin{aligned}
(u) & =\operatorname{det} \frac{\partial\left(u_{1}, \cdots, u_{n}\right)}{\partial\left(x_{1}, \cdots, x_{n}\right)}=\frac{\operatorname{det}\left(X^{i} u_{j}\right)}{h} \\
& =\frac{\exp \left(-\sum_{i=1}^{n} \omega_{i}{ }^{i}\right)}{h}=c \prod h_{j}^{-g-1}=0
\end{aligned}
$$

Hence the map $u$ is locally homeomorph.

Let $g$ be an element of $\pi_{1}\left(C^{n}-D\right)$ and $u$ be a solution in $U$. The analytic continuation of $u$ along $g$ gives another solution $v$ of $U_{\nabla}$. Thus $\pi_{1}\left(C^{n}-D\right) \ni g$ operates on $U_{v}$ as a linear endmorphism $v=\rho(g) u$. We call the representation

$$
\rho: \pi_{1}\left(C^{n}-D\right) \rightarrow G L\left(U_{\nabla}\right)
$$

the monodromy representation of $\nabla$. Let us denote by $\widetilde{x}=\tilde{x} g$ the action of $g \in \pi_{1}\left(\boldsymbol{C}^{n}-D\right)$ as a covering transformation of $\widetilde{C^{n}-D}$. Then we have $u(\tilde{x} g)=u(x) \rho(g)$.

The inditial equation for an irreducible component $D_{k}$ of $D$ is given by $I_{k}(\lambda)=\operatorname{det}\left(\lambda \delta_{j}{ }^{i}-\right.$ residue $\left._{D_{k}}\left(\omega_{\jmath}{ }^{i}\right)\right)=0$, whose roots give the exponents of the monodromy of the path turning once around the generic point of $D_{k}$. 
Now let $P=P\left(x_{1}, \cdots, x_{n}, \frac{\partial}{\partial x_{1}}, \cdots, \frac{\partial}{\partial x_{n}}\right)$ be a differential operator which is homogeneous of degree $k$ with respct to the previous graduation. Then we have a formula: $\left[X^{1}, p\right]=X^{1} P-P X^{1}=k P$, where $X^{1}=\sum_{i=1}^{n} m_{i} x_{i}$ $\times \frac{\partial}{\partial x_{i}}$.

On the other hand $P_{j k}=X^{k} X^{j}+\sum_{i=1}^{n} P_{i}^{j k} X^{i}$ is homogeneous of degree $d_{k}+d_{j}$. Thus the correspondence $u \mid \rightarrow X^{1} u$ defines an endmorphism $B$ : $U_{\nabla} \rightarrow U_{\nabla}$

By integrating $\left(X^{1} u_{1}, \cdots, X^{1} u_{n}, X^{1} 1\right)=\left(u_{1}, \cdots, u_{n}, 1\right) B$, we have the weighted homogenity of the map $u$.

$$
u\left(t^{m_{1}} x_{1}, \cdots, t^{m_{n}} x_{n}\right)=u\left(x_{1}, \cdots, x_{n}\right) \exp (B \log t)
$$

One of the uniformizaticn equations

$$
X^{1} X^{1} u+\Gamma_{1}^{11} X^{1} u=0
$$

implies that the minimal polynomial of $B$ must be a factor of the polynomial $\lambda^{2}+\Gamma_{1}{ }^{11} \lambda=\lambda\left(\lambda+\Gamma_{1}{ }^{11}\right)$. Hence the eigenvalues of $B$ can be zero or $-\Gamma_{1}^{11}$.

By choosing a suitable basis of $U_{\nabla}, B$ has the form

$$
\text { i) }\left[\begin{array}{rrrrr}
-\Gamma_{1}^{11} & & & \\
& \ddots & & 0 & \\
& & -\Gamma_{1}^{11} & \\
& 0 & 0 & \\
& & & \ddots & 0
\end{array}\right]
$$$$
\text { ii) }\left[\begin{array}{cccccc}
0 & & & & \\
10 & & & & \\
& 0 & & & \\
& 10 & & & \\
& & \ddots & & \\
& & & 0 & \\
& & & & \ddots & 0 \\
& & & & 0
\end{array}\right]
$$

The restriction of $B$ to $U_{\nabla} / C$ is conjugate to the matrix $\left(\Gamma_{i}^{1, j}\right)_{i, j=1, \ldots, n}$. Especially in the case $i$ ), the solution space $U_{\nabla}$ is a direct sum of the space of functions homogeneous of degree $-\Gamma_{1}^{11}$ and the space of functions homogeneous of degree 0 .

\section{§ 3. The Inversion Problem}

Let us denote by $H_{\nabla}$ the image domain of the map $u: \overparen{C^{n}-D \rightarrow V^{*}}$. In general there does not exist a univalent mapping $E: H \rightarrow C^{n}-D$ so that 


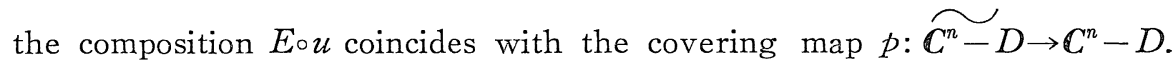
We will study a rather delicate problem concerned with the existence of such a map.

For this purpose let us introduce a concept of the b-b-map.

Definition. A multi-valued map $w: \mathbb{C}^{n}-D \rightarrow H \subset \mathbb{C}^{m}$ is a b-b-map if it satisfies the following condition; For any discrete subset of $\mathbb{C}^{n}-D$, the image of $A$ by $w, \widetilde{w}\left(p^{-1}(A)\right)$ is a discrete subset of $H$. Here

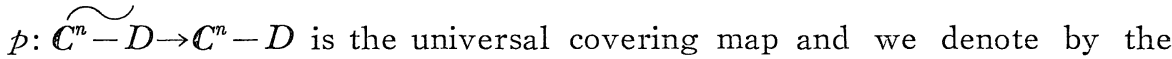
$\widetilde{w}$ the lifting of $w$ to $\overparen{C^{n}-D \rightarrow H}$.

Roughly speaking, this condition ensures that the boundary of $C^{n}-D$ should correspond to the boundary of $H$ by $w$. For instance $z^{\alpha}: \mathbb{C}-\{0\}$ $\rightarrow \mathbb{C}-\{0\}$ is a b-b-map iff $\alpha$ is a rational number.

We notice that if $u_{\nabla}$ is a b-b-map, then the orbit by the monodromy $u^{*} \rho\left(\pi_{1}\left(\mathbb{C}^{n}-D\right)\right)$ is a discrete subset of $H$ for $u^{*} \in H$, hence $\rho\left(\pi_{1}\left(\mathbb{C}^{n}-D\right)\right)$ is a discrete subset of $G L\left(V_{\nabla}\right)$.

Furthermore assuming that $u_{\nabla}$ is a b-b-map, we conclude the following:

I. $\rho \pi_{1}\left(\mathbb{C}^{n}-D\right)$ acts on $H_{\nabla}$ in properly discontinuous fashon.

Let us denote by $W_{\nabla}$ the quotient space of $H_{\sigma}$ by $\rho \pi_{1}\left(\boldsymbol{C}^{n}-D\right)$ and let $E: H_{\nabla} \rightarrow W_{\nabla}$ be the quotient map.

II. The reduction of $u_{\nabla}$ to

$$
C^{n}-D / \operatorname{ket} \rho \rightarrow H_{\nabla}
$$

is a finite covering map.

III. There exists a proper finite map $\mathbb{C}^{n}-D \rightarrow W_{\nabla}$ so that the diagram below commutes.

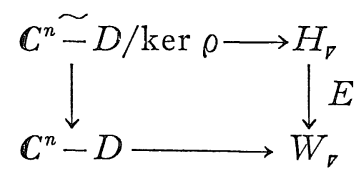


Conversely if $u_{\nabla}$ satisfies the above three conditions I, II, III, it is a b-b-map.

Let $q$ be the sheet number of the covering $C^{n}-D / \operatorname{ker} \rho \rightarrow H_{\nabla}$. Then for a point $u^{*} \in H_{\nabla}$, we have the following formula.

$$
q=p\left(u^{-1}\left(u^{*}\right)\right) \cdot \text { isotropy subgroup of } u^{*} \text {. }
$$

This shows especially $\boldsymbol{C}^{n}-D \rightarrow W$ is an unramified covering iff $\rho \pi_{1}\left(C^{n}-D\right)$ operates fixed-point-freely on $H_{\nabla}$. The covering $C^{n}-D \rightarrow W$ is normal iff $\pi_{1}(H)$ is a normal subgroup of $\pi_{1}(H) \pi_{1}\left(\boldsymbol{C}^{n}-D\right)$.

Now among all the boundary points of $H$ let us distinguish some special points.

Definition. $R_{\nabla}=\left\{u^{*} \in \partial H\right.$; there exists a path $\varphi:\left([01) \rightarrow C^{n}-D\right.$

$$
\begin{aligned}
& \text { such that } \lim _{t \rightarrow 1} \varphi(t) \subset D \text {, and for a lifting } \\
& \left.\tilde{\varphi}:[01) \rightarrow C^{n}-D, u^{*}=\lim _{t \rightarrow 1} u_{\nabla}(\widetilde{\varphi}(t))\right\} .
\end{aligned}
$$

\section{Problems}

I. Is $R_{\nabla}$ a dense subset of $\partial H_{\nabla}$, which can be stratified by open subsets of linear subspaces of $V_{\nabla}{ }^{*}$ of the form $\operatorname{Im}\left(\sum a_{i} u_{i} / \sum b_{i} u_{i}\right)>0$ ? Here $u_{1}, \cdots, u_{n}$ is a basis for $V_{\nabla}$.

II. Suppose $R_{\nabla}$ has a stratum corresponding to a hypersurface defined by $u=0$ for a $u \in V_{\nabla}$. By the monodromy invariantness of $R_{\nabla}, R_{\nabla}$ contains strata of $\rho \pi_{1}\left(C^{n}-D\right) u$. Let us consider the series: $\varphi_{u, s}=\sum_{v \in \rho \pi_{1}\left(\boldsymbol{C}^{n}-D\right) u} v^{-s}$. Does it converge for $\operatorname{Re}(s) \gg 0$ on an $H_{\nabla}$, and cit be analytically continuable on the whole plane for $s$ ?

III. Can those solutions $u$ defining strata of $R_{\nabla}$ be characterized as some special functions along Lagrangean variety $L(\log D)$ so that the meromorphic function field of $W_{\nabla}$ is generated by $\varphi_{u_{j}, s_{k}}$ for some representatives $u_{j}$ corresponding to an irreducible component $L_{j}$ of $L(\log D)$ and complex numbers $s_{k}$ ?

For the moment we do not have any general answer to the problems. 
There are some classical examples of interest.

i) For $n=2$ and $D=\{x y(x-y)=0\}$, the system of the uniformizaion equations reduce to Gegenbauer's differential equations and hypergeometric differential equations, where we have a well developed theory due to Schwarz and Christoffel.

ii) For $n=2$ and $D=\left\{27 g_{3}{ }^{2}-g_{2}{ }^{3}=0\right\}$, we get elliptic modular functions and equilateral triangle function.

iii) For $n=3$ and $D=$ the discriminant for the Weyl group of type $A_{3}$, some example is discussed in the note [2], where some modification of the above problems are positively solved.

\section{References}

[1] Bourbaki, N., Groupes et algèbres de Lie, Chapitres 4, 5 et 6. Hermann, Paris, 1969.

[2] Saito, K., On the uniformization of complements of discriminant loci, A. M. S. Summer Institute 1975. 
